doi:10.1103/PhysRevB.84.092106

\title{
Predicting dislocation climb: Classical modeling versus atomistic simulations
}

\author{
Emmanuel Clouet* \\ Service de Recherches de Métallurgie Physique, CEA/Saclay, 91191 Gif-sur-Yvette, France
}

(Dated: August 20, 2021)

\begin{abstract}
The classical modeling of dislocation climb based on a continuous description of vacancy diffusion is compared to recent atomistic simulations of dislocation climb in body-centered cubic iron under vacancy supersaturation [Phys. Rev. Lett. 105, 095501 (2010)]. A quantitative agreement is obtained, showing the ability of the classical approach to describe dislocation climb. The analytical model is then used to extrapolate dislocation climb velocities to lower dislocation densities, in the range corresponding to experiments. This allows testing of the validity of the pure climb creep model proposed by Kabir et al. [Phys. Rev. Lett. 105, 095501 (2010)].

PACS numbers: 61.72.Lk, 62.20.Hg
\end{abstract}

Dislocations can move out of their glide planes through the emission or absorption of point defects. Such a mechanism, known as dislocation climb, has been modeled for more than half a century now using a continuum description of matter and diffusion theory ${ }^{1,2}$. One can thus find in most of the textbooks on dislocations ${ }^{3-7}$ analytical expressions which give the dislocation climbing velocity as a function of the applied stress, the temperature, the point defect supersaturation, etc. Although this classical description has been shown to reasonably explain experimental observations $\mathrm{s}^{\frac{8}{13}}$, a quantitative validation by direct comparison to experiments is out of reach. Dislocation climb is indeed rarely the single mechanism producing plastic strain and one has usually to deal with a complex dislocation microstructure.

On the other hand, atomistic simulations can be used to study the pure climb either of an isolated dislocation or of a well controlled dislocation microstructure. Such simulations thus offer a natural way for a quantitative validation of the classical approach. In this Brief Report, we compare predictions of the classical dislocation climb mode ${ }^{3-7}$ to the results of atomistic simulations published by Kabir et al. in Ref. 14 .

Kabir et al.$\underline{14}$ performed atomistic simulations to study the climb of a mixed dislocation in body-centered cubic iron. Their simulations are based on a kinetic Monte Carlo algorithm which reproduces the diffusion of vacancies and their annihilation on a dislocation jog. The influence of the dislocation on the vacancy migration barriers is fully taken into account thanks to an empirical potential which allows them to search for the minimum energy path joining all neighboring vacancy stable positions (nudged elastic band method). In these simulations, the dislocations can be considered as being saturated with jogs because of the small dislocation length, and the climb is driven by a high vacancy supersaturation.

When pipe diffusion is fast enough and a high concentration of jogs is present on the dislocation, one can assume that vacancies are at equilibrium all along the

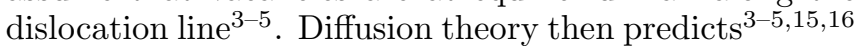

that an infinite straight dislocation of Burgers vector $\mathbf{b}$ and character $\theta$ climbs at a velocity

$$
v_{\mathrm{cl}}=\eta \frac{D_{\mathrm{V}}}{b|\sin (\theta)| \ln \left(R_{\infty} / r_{\mathrm{c}}\right)}\left|C_{\mathrm{V}}^{\mathrm{eq}}-C_{\mathrm{V}}^{\infty}\right| .
$$

The geometric factor $\eta=2 \pi$ for an isolated dislocation. $D_{\mathrm{V}}$ is the vacancy diffusion coefficient in a dislocationfree crystal, $C_{\mathrm{V}}^{\mathrm{eq}}$ the concentration of vacancies in equilibrium with the dislocation at a distance $r_{\mathrm{c}} \sim b$ from the line, and $C_{\mathrm{V}}^{\infty}$ the average vacancy concentration imposed at a distance $R_{\infty}$. This should correspond to half the average distance between dislocations, i.e. $R_{\infty}=1 /\left(2 \sqrt{\rho_{\mathrm{D}}}\right)$ if $\rho_{\mathrm{D}}$ is the dislocation density. For a high vacancy supersaturation, like in the atomistic simulations of Kabir et al. $\stackrel{14}{\underline{\mathrm{e}}}, C_{\mathrm{V}}^{\mathrm{eq}} \ll C_{\mathrm{V}}^{\infty}$ and then

$$
v_{\mathrm{cl}}=-\eta \frac{D_{\mathrm{V}}}{b|\sin (\theta)| \ln \left(2 r_{\mathrm{c}} \sqrt{\rho_{\mathrm{D}}}\right)} C_{\mathrm{V}}^{\infty} .
$$

Equation (11) predicts that the climb velocity, once normalized by $D_{\mathrm{V}} C_{\mathrm{V}}^{\infty}$, should only depend on $\rho_{\mathrm{D}}$. This agrees with the atomistic simulations of Ref. 14: Results for all temperatures and vacancy supersaturations are well reproduced by Eq. (11). (Figs. 1) and 2). The two parameters $\eta$ and $r_{\mathrm{c}}$ appearing in this equation were used as fitting parameters, and the best quantitative agreement with atomistic simulations was obtained for the values $\eta=12.8$ and $r_{\mathrm{c}}=4.3 b$.

The geometric factor obtained from this fit $(\eta \sim 4 \pi)$ is close to its $2 \pi$ theoretical value. The slight difference may come from the fact that a periodic array of dislocation dipoles has been modeled in the atomistic simulations of

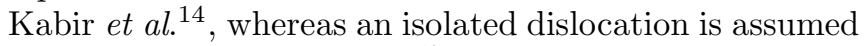
in Eq. (1). Burke and Nix ${ }^{17}$ also showed that the elastic interaction between the vacancy and the dislocations, which is neglected in our modeling approach, leads to a value for $\eta$ slightly higher than $2 \pi$, thus in agreement with what we obtained.

The conventions are usually to take the capture radius $r_{\mathrm{c}}$ equal to the norm of the Burgers vector. Our fit of Eq. (1) leads to $r_{\mathrm{c}}=4.3 \mathrm{~b}$, which actually agrees with 


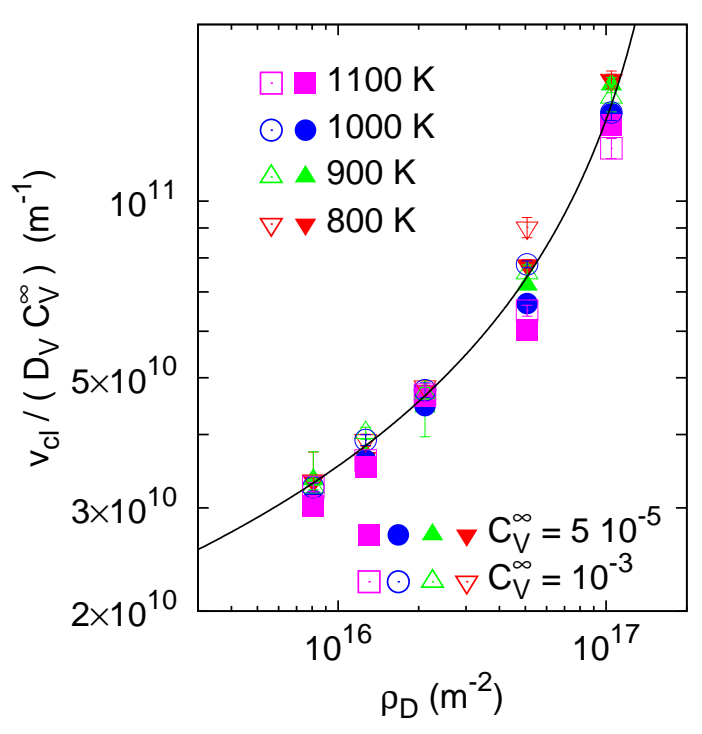

FIG. 1. Variation with the dislocation density $\rho_{\mathrm{D}}$ of the climb velocity $v_{\mathrm{cl}}$ normalized by $D_{\mathrm{V}} C_{\mathrm{V}}^{\infty}$ for different temperatures and vacancy supersaturations. Symbols corresponds to atomistic simulations ${ }^{14}$ and lines to Eq. (1).

the definition of the core region $(r<4 b)$ that has been deduced from previous atomistic calculations $\frac{18}{}$ on the same model system.

One therefore sees that the two parameters $\eta$ and $r_{\mathrm{c}}$ are physical parameters whose precise values are close to theoretical ones. It is also important to notice that both parameters do not theoretically depend on the dislocation density, the vacancy supersaturation, the applied stress, nor the temperature. This was checked and all atomistic simulations of Ref. 14 could be reproduced with a single set of parameters.

This comparison with atomistic simulations shows that the classical modeling of dislocation climb leads to quantitative predictions. Such a model, based on a continuous description of vacancy diffusion, does not explicitly take into account all atomic details of the vacancy diffusion close to the dislocation. It nevertheless manages to perfectly reproduce results of atomistic simulations. Finally, all atomistic details on the vacancy migration are not so relevant to model dislocation climb and one can use classical mesoscopic approaches ${ }^{3-7,15,16}$ leading to simple analytical expressions like Eq. (11).

One can now use this expression of dislocation climb mobility to compare predictions of different creep models with experimental data, in particular the creep model developed by Kabir et al. ${ }^{14}$. The dislocation densities in the atomistic simulations $\frac{14}{\underline{4}}\left(\rho_{\mathrm{D}} \gtrsim 10^{16} \mathrm{~m}^{-2}\right)$ are much higher than the ones usually observed in creep experiments in iron $\frac{19,20}{20}\left(\rho_{\mathrm{D}} \lesssim 10^{12} \mathrm{~m}^{-2}\right)$. As a consequence, one cannot directly use the dislocation climb velocities measured in atomistic simulations, but one needs to extrapolate them to lower dislocation densities. Kabir et al. $\stackrel{14}{\underline{4}}$ used power laws to perform such an extrapolation and concluded to the agreement of their model with creep experiments. As such power laws do not rely on any physical ground, it is worth checking if the same nice agreement can be obtained when the classical modeling of dislocation climb leading to Eq. (1) is used for this extrapolation.

In the creep model, one usually uses a power law $v_{\mathrm{cl}} \propto$ $\rho_{\mathrm{D}}{ }^{m}$ to reproduce the variations of the climbing velocity with the dislocation density. Equation (1) leads then to an exponent $m$ which depends on the density $\rho_{\mathrm{D}}$

$$
m=\frac{\partial \ln \left(v_{\mathrm{cl}}\right)}{\partial \ln \left(\rho_{\mathrm{D}}\right)}=\frac{-1}{2 \ln \left(2 r_{\mathrm{c}} \sqrt{\rho_{\mathrm{D}}}\right)} .
$$

This exponent can now be used in the creep model pro-

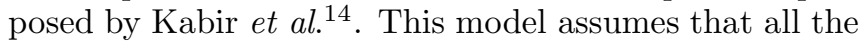
plastic strain in creep is produced by climbing dislocations. Orowan law gives then the creep rate $\dot{\varepsilon}=\rho_{\mathrm{D}} b v_{\mathrm{cl}}$. The dislocation density is fixed by the Taylor relation $\left(\rho_{\mathrm{D}}=(\sigma / \alpha G b)^{2}\right.$ where $\alpha \sim 0.4$ is an empirical constant 14 and $G$ is the shear modulus). The vacancy supersaturation, which varies linearly both with the applied stress $\sigma$ and the dislocation velocity, is given by Eq. (1) in Ref. 14 . Equation (2) combined with these assumptions leads then to a steady-state creep rate whose stress and temperature dependences can be reproduced by the power law $\dot{\varepsilon}=\mathcal{A} \sigma^{n} \exp (-Q / k T)$ with a stress-dependent exponent $n=3+4 m$ :

$$
n(\sigma)=3-\frac{2}{\ln \left(2 r_{\mathrm{c}} \sigma / \alpha G b\right)}
$$

Equation (3) allows applying the creep model of Kabir et al. to a stress range much lower than in atomistic simulations. Stress in creep experiments in iron usually does not exceed $100 \mathrm{MPa}^{19-24}$. Equation (3) then predicts an exponent $n$ smaller than 3.5, thus far from the values higher than 6 found experimentally 23,24 . One therefore sees that the creep model proposed by Kabir et al. cannot explain experimental stress exponents measured in iron. It naturally leads at low stress to an exponent close to 3 , like any other creep model based on pure climb 25 . The high value for $n$ obtained by Kabir et al. ${ }^{14}$ corresponds to the much higher dislocation density of their atomistic simulations and cannot be directly compared to these experimental values.

One probably needs to consider both dislocation glide and climb to obtain a stress exponent close to the experimental one. A creep model based on dislocation pure climb is only valid when dislocations cannot glide because of some constraints, crystallographic constraints for instance as in hcp metals 26,27 or quasicrystals ${ }^{13,28}$. Creep in alpha iron is far from this ideal case as there is nothing preventing the dislocations from gliding. As pointed out by Weertman in his review paper ${ }^{29}$, "almost all of the creep strain is produced by glide motion of dislocations". Although dislocation climb is the rate limiting process, dislocation glide strongly affects creep and cannot be ignored. Weertman $25,29,30$ showed for instance that the consideration of glide in a creep model makes the stress exponent increase from $n=3$ to $n=4.5$. 

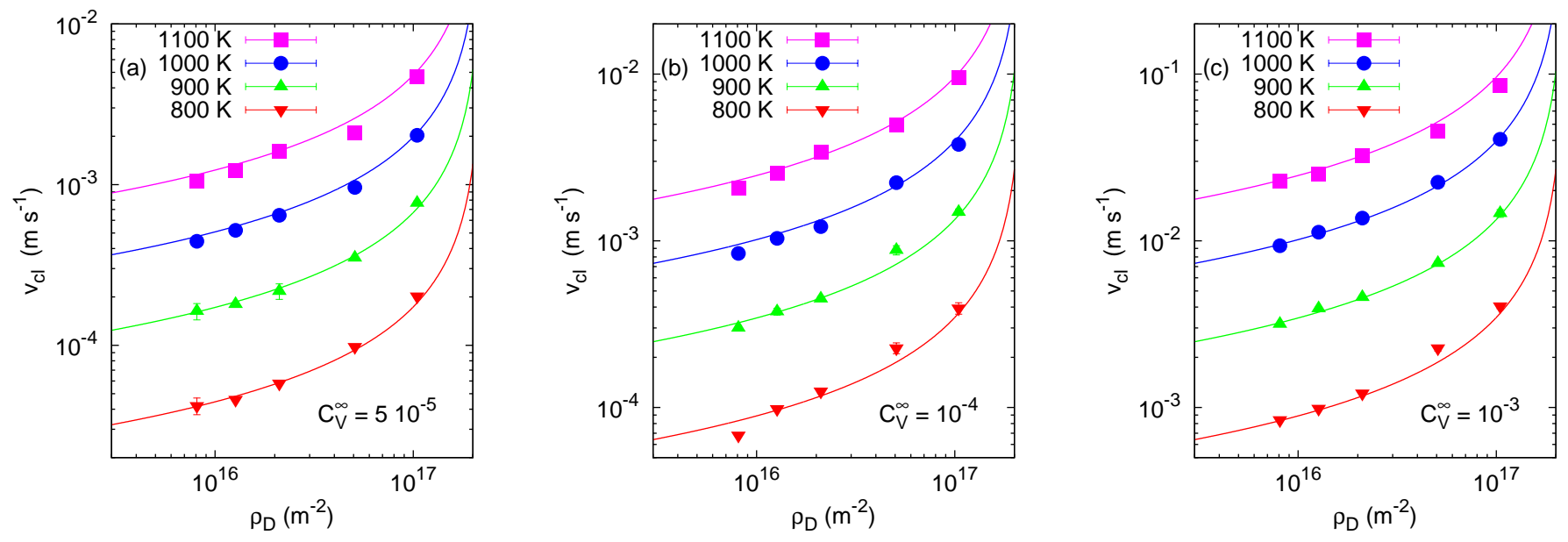

FIG. 2. Variation with the dislocation density $\rho_{\mathrm{D}}$ of the climb velocity $v_{\mathrm{cl}}$ for different temperatures and for a vacancy concentration (a) $C_{\mathrm{V}}^{\infty}=510^{-5}$, (b) $C_{\mathrm{V}}^{\infty}=10^{-4}$, and (c) $C_{\mathrm{V}}^{\infty}=10^{-3}$. Symbols corresponds to atomistic simulations ${ }^{14}$ and lines to Eq. (1).

Another discrepancy between the creep model of Kabir et al. ${ }^{14}$ and experiments $\frac{21-23}{2}$ in iron comes from the temperature dependence of the stress exponent $n$ and the stress dependence of the creep activation energy $Q$. According to Eq. (3), $n$ does not depend directly on the temperature. A temperature dependence may only arise through a variation of the capture radius $r_{\mathrm{c}}$ with the temperature ${ }^{31}$. As shown above, a single value of $r_{\mathrm{c}}$ could be used to reproduce all the dislocation climbing velocities obtained by atomistic simulations in the temperature range $800-1100 \mathrm{~K}$. As a consequence, this creep model does not lead to any temperature dependence of the stress exponent $n$. The creep rate is obtained from Orowan law $\dot{\varepsilon}=\rho_{\mathrm{D}} b v_{\mathrm{cl}}$ where the dislocation density is deduced from Taylor relation and the climbing velocity is given by Eq. (1). The activation energy $Q$ for creep is then the activation energy for vacancy diffusion whatever the applied stress. The dependences found by Kabir et al. (Fig. 4 in Ref. 14) are artifacts caused by a fit of the climbing velocity at high densities using a simple power law which neglects the logarithm appearing in Eq. (11). To explain the dependence observed experimentally $21-23$, one has therefore to rely on mechanisms which are not considered in the creep model of Kabir et al..

Finally, it is worth pointing that the stress enters in the creep model of Kabir et al. $\stackrel{14}{\underline{1}}$ only through the control of the dislocation density and of the vacancy supersaturation. As the climbing velocity does not depend then on the dislocation orientation, all dislocations, whatever their orientations, are climbing at the same velocity. If there is no specific climbing direction being enhanced by the stress, no average macroscopic strain can develop and there will be no creep.

In summary, the comparison with atomistic simulations shows that a classical approach at a mesoscopic scale manages to quantitatively describe dislocation climb. Such an approach not only allows rationalizing results of atomistic simulations, but it is also necessary to extrapolate them in a range of dislocation densities corresponding to experiments. Thanks to such an extrapolation based on a physical sound model, a fair test of the validity of the creep model proposed by Kabir et $a l . \underline{14}$ can be made, thus showing its inability to reproduce experimental data on creep in iron.
* emmanuel.clouet@cea.fr

1 N. F. Mott, Proc. Phys. Soc. B, 64, 729 (1951).

2 J. Weertman, J. Appl. Phys., 26, 1213 (1955).

3 J. Friedel, Dislocations (Pergamon Press, Oxford, 1964).

4 J. P. Hirth and J. Lothe, Theory of Dislocations, 2nd ed. (Wiley, New York, 1982).

5 D Caillard and J. L. Martin, Thermally Activated Mechanisms in Crystal Plasticity, edited by R. W. Cahn (Pergamon, Amsterdam, 2003).

${ }^{6}$ F. R. N. Nabarro, Theory of Crystal Dislocations (Oxford Uhiv. Press, London, 1967).

7 V. L. Indenbom and Z. K. Saralidze, in Elastic Strain
Fields and Dislocation Mobility, Modern Problems in Condensed Matter Sciences, Vol. 31, edited by V. L. Indenbom and J. Lothe (North-Holland, Amsterdam, 1992) Chap. 10, pp. 571-624.

8 J. Silcox and M. J. Whelan, Philos. Mag. 5. 1 (1960)

9 D. N. Seidman and R. W. Balluffi, Phys. Status Solidi, 17, $531(1966)$

${ }^{10}$ R. W. Balluffi, Phys. Status Solidi B, 31, 443 (1969).

11 J. Powell and J. Burke, Philos. Mag., 31, 943 (1975).

12 C. Bonafos, D. Mathiot, and A. Claverie, J. Appl. Phys., 83, 3008 (1998).

${ }^{13}$ F. Mompiou and D. Caillard, Acta Mater., 56, 2262 (2008). 
14 M. Kabir, T. T. Lau. D. Rodney, S. Yip, and K. J. Van Vliet, Phys. Rev. Lett., 105, 095501 (2010).

15 D. Mordehai, E. Clouet, M. Fivel, and M. Verdier, Philos. Mag., 88, 899 (2008).

16 B. Bakó, E. Clouet, L. M. Dupuy, and M. Blétry, Philos. Mag., 91, 3173 (2011).

17 M. A. Burke and W. D. Nix, Philos. Mag. A, 37, 479 (1978).

18 T. T. Lau, X. Lin, S. Yip, and K. J. Van Vliet, Scripta Mater., 60, 399 (2009).

19 F. Garofalo, L. Zwell, A. Keh, and S. Weissmann, Acta Metall., 9, 721 (1961)

20 S. Karashima, T. Iikubo, T. Watanabe, and H. Oikawa, Trans. Jpn. Inst. Met., 12, 369 (1971).

21 S. Karashima, H. Oikawa, and T. Watanabe, Acta Metall. 14, $791(1966)$.

22 J. Cadek and K. Milicka, Czech. J. Phys., 18, 1156 (1968).

23 J. Cadek, M. Pahutova, K. Cíha, and T. Hostinsky, Acta Metall., 17, 803 (1969).
${ }^{24}$ P. W. Davies, G. Nelmes, K. R. Williams, and B. Wilshire, Met. Sci. J., 7, 87 (1973).

25 J. Weertman, in Rate Processes in Plastic Deformation of Metals, edited by J. C. M. Li and A. K. Mukherjee (Am. Soc. Metals, Metals Park, Ohio, 1975) pp. 315-336.

26 G. Edelin and J. P. Poirier, Philos. Mag., 28, 1203 (1973).

27 R. Le Hazif, G. Edelin, and J. M. Dupouy, Metall. Mater. Trans. B, 4, 1275 (1973).

${ }^{28}$ F. Mompiou and D. Caillard, Mater. Sci. Eng. A, 483-484, 143 (2008).

29 J. Weertman, ASM Transactions Quarterly, 61, 681 (1968), ISSN 0097-3912.

30 J. Weertman, Phys. Rev., 107, 1259 (1957).

31 The temperature dependence of the shear modulus $G$ is taken into account by normalizing the applied stress $\sigma$ with $G$ when processing results of creep experiments. A temperature dependence of the parameter $\alpha$ entering Taylor relation is also possible but the modeling of such a variation will require a more complex approach than the simple creep model used here. 